PROCEEDINGS OF THE

AMERICAN MATHEMATICAL SOCIETY

Volume 132, Number 11, Pages 3327-3336

S 0002-9939(04)07473-8

Article electronically published on June 22, 2004

\title{
SUBMANIFOLDS ASSOCIATED WITH GRAPHS
}

\author{
ALFONSO CARRIAZO AND LUIS M. FERNÁNDEZ
}

(Communicated by Mohan Ramachandran)

\begin{abstract}
In this paper we present an interesting relationship between graph theory and differential geometry by defining submanifolds of almost Hermitian manifolds associated with certain kinds of graphs. We show some results about the possibility of a graph being associated with a submanifold and we use them to characterize $\mathrm{CR}$-submanifolds by means of trees. Finally, we characterize submanifolds associated with graphs in a four-dimensional almost Hermitian manifold.
\end{abstract}

\section{INTRODUCTION}

An important research subject in the theory of submanifolds of an almost Hermitian manifold is the study of a submanifold from the point of view of its behaviour with respect to the almost complex structure. Many submanifolds are characterized by such a homogeneous behaviour: complex submanifolds, totally real submanifolds, CR-submanifolds, and, more recently, slant or semi-slant submanifolds. In this paper, we will show that, in all of these cases, we can find a graph closely related to the algebraic structure on the submanifold.

The idea of the association between the above stated submanifolds and the corresponding graphs emerges from the study of slant surfaces. Given a proper $\theta$-slant surface $M^{2}$ in a 4 -dimensional almost Hermitian manifold $\left(\widetilde{M}^{4}, J, g\right)$, it was shown by Chen in [1] that we can consider a special local orthonormal frame, namely an adapted slant frame, $\left\{X_{1}, X_{2}, X_{3}, X_{4}\right\}$ such that $X_{1}, X_{2}$ are tangent to $M, X_{3}, X_{4}$ are normal to $M$ and they satisfy the following equalities:

$$
g\left(J X_{1}, X_{2}\right)=-g\left(J X_{3}, X_{4}\right)=\cos \theta, \quad g\left(J X_{1}, X_{3}\right)=g\left(J X_{2}, X_{4}\right)=\sin \theta,
$$

It is clear that (1.1) completely determines the behaviour of the almost complex structure $J$ on $M$. Then, it is interesting to give a graphic expression of the above relationships. We can easily define a graph by using the following method. We first consider a vertex for every field of the adapted slant frame by labeling each vertex with its corresponding natural index. In this case, we obtain the set of vertices $\{1,2,3,4\}$. Now, we say that the $\{i, j\}$ edge exists if and only if $g\left(J X_{i}, X_{j}\right) \neq 0$.

Received by the editors July 25, 2001.

2000 Mathematics Subject Classification. Primary 53C40, 05 C90.

Key words and phrases. Almost Hermitian manifold, CR-submanifold, slant submanifold, graph theory.

The authors were partially supported by the PAI project (Junta de Andalucía, Spain, 2002).

(C)2004 American Mathematical Society 
Note that, since $g\left(J X_{i}, X_{j}\right)=-g\left(J X_{j}, X_{i}\right)$, the definition of the existence of an edge is not ambiguous. Hence, we have Graph 1 in Figure 1, Moreover, we can assign a weight on every edge. It is reasonable to consider on $\{i, j\}$ the weight given by $g\left(J X_{i}, X_{j}\right)$, but it is not possible because the edge does not depend on the order of its vertices. Therefore, we consider $g^{2}\left(J X_{i}, X_{j}\right)$ as the weight of $\{i, j\}$, and we have Graph 2 shown in Figure 1 .
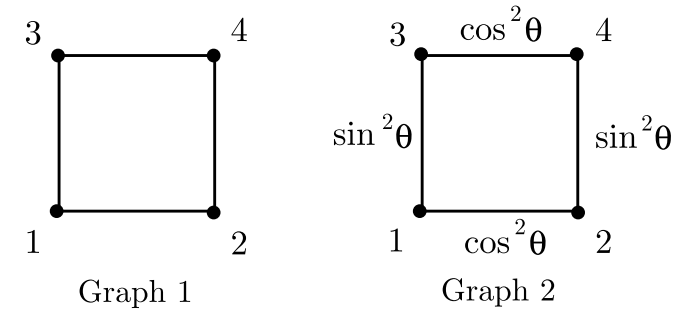

Figure 1. Graph associated with a slant surface.

Note that we obtain additional visual information by putting the vertices corresponding to tangent fields at an imaginary bottom line and those which correspond to normal fields at a top line.

In this paper, we generalize the above situation by defining submanifolds associated with graphs. Basically, we first construct a graph related to the tangent space at an arbitrary point of a submanifold, and then, we say that this submanifold is associated with the graph if it can be, in a certain way, differentiably extended to every point of the submanifold.

We show some results about the possibility of a graph being associated with a submanifold and we use them to characterize CR-submanifolds by means of trees (connected graphs without cycles). We also characterize slant surfaces. Finally, we study and characterize submanifolds associated with graphs in a four-dimensional almost Hermitian manifold.

We think that the appropriate analysis of the association between these two different objects (the submanifolds from the Complex Geometry and the graphs from the Discrete Mathematics) will provide us with a new tool which can be useful in studying the general problem of the classification of submanifolds. Later, it establishes a new link between two traditionally remote research areas.

\section{Preliminaries}

In this section, we first recall some basic formulas and definitions about slant submanifolds in Complex Geometry, which we shall use later. For details and background on complex manifolds, we refer to the standard reference [4].

A submanifold $M$ of an almost Hermitian manifold $(\widetilde{M}, g, J)$ is said to be slant [1] if for each nonzero vector $X$ tangent to $M$ at $p$, the angle $\theta(X), 0 \leq \theta(X) \leq \pi / 2$, between $J X$ and $T_{p} M$ is a constant, called the slant angle of the submanifold. In particular, complex and totally real submanifolds appear as slant submanifolds with slant angle 0 and $\pi / 2$, respectively. A slant submanifold is called proper slant if it is neither complex nor totally real. Put $J X=P X+F X$, for any tangent vector field $X$, where $P X$ (resp. $F X$ ) denotes the tangential (resp. normal) component of $J X$. Then, $\theta$-slant submanifolds are characterized by the formula $P^{2}=-\cos ^{2} \theta I d$. 
Similarly, a differentiable distribution $\mathcal{D}$ on $M$ is said to be a slant distribution if for any nonzero vector $X \in \mathcal{D}_{p}$, the angle between $J X$ and the vector space $\mathcal{D}_{p}$ is constant, that is, it is independent of the choice of $p \in M$ and of $X \in \mathcal{D}_{p}$. This constant angle is called the slant angle of the slant distribution $\mathcal{D}$. By using this definition, Papaghiuc introduced the notion of a semi-slant submanifold of an almost Hermitian manifold 3. A submanifold $M$ is said to be a semi-slant submanifold if there exist on $M$ two differentiable orthogonal distributions $\mathcal{D}_{1}$ and $\mathcal{D}_{2}$ such that $T M=\mathcal{D}_{1} \oplus \mathcal{D}_{2}, \mathcal{D}_{1}$ is a complex distribution $\left(J\left(\mathcal{D}_{1}\right)=\mathcal{D}_{1}\right)$ and $\mathcal{D}_{2}$ is a slant distribution with the slant angle $\theta \neq 0$. This notion clearly generalizes those of both slant and CR submanifolds.

On the other hand, a graph $G$ is a pair $(V, A)$, where $V$ is a finite nonempty set of vertices and $A$ is a prescribed set of unordered pairs of distinct vertices of $V$. Given a pair $\{i, j\}$ in $A, i$ and $j$ are said to be adjacent vertices and $\{i, j\}$ is said to be incident with both $i$ and $j$. The degree of a vertex is the number of edges incident with it. The graph consisting of just two vertices and one edge between them is called $K_{2}$.

A graph $G$ is labeled when the vertices are distinguished from each other by names. In this paper, we are labeling graphs by using consecutive natural numbers. Therefore, we consider that the vertex set of a graph with $n$ vertices is just $\{1, \ldots, n\}$. Moreover, we are working on weighted graphs, i.e., graphs such that every edge has an assigned weight (a real number).

An isomorphism between two graphs is a one-to-one correspondence between their vertex sets which preserves adjacency. So, it is obvious that the vertex numbers of two isomorphic graphs must be the same. Given that we are considering weighted labeled graphs, we also impose from now on that isomorphisms preserve labels and weights. Therefore, throughout this paper, an isomorphism between two graphs with $n$ vertices is just the identity map from $\{1, \ldots, n\}$ into itself, preserving adjacency and edge weights. For more background on graph theory, we refer to [2].

\section{SUbmanifoldS ASSOCiated With GRAPHS}

Let $M^{m}$ be a Riemannian manifold isometrically immersed in an almost Hermitian manifold $\left(\widetilde{M}^{n}, J, g\right)$. Let $\mathcal{B}=\left\{X_{1}, \ldots, X_{n}\right\}$ be a local orthonormal frame defined on a neighbourhood $U$ of a point $p \in M$. Then, for any $q \in U$, we define the weighted graph $G_{\mathcal{B}, q}$ given by the set of vertices $\{1, \ldots, n\}$ such that the edge $\{i, j\}$ exists if and only if $g_{q}\left(J_{q} X_{i q}, X_{j q}\right) \neq 0$, with weight $g_{q}^{2}\left(J_{q} X_{i q}, X_{j q}\right)$. Note that this is just a generalization of the initial construction procedure.

Now we can introduce the idea of association between submanifolds and graphs. Let $M^{m}$ be a submanifold of an almost Hermitian manifold $\left(\widetilde{M}^{n}, J, g\right)$ and let $G$ be a weighted graph with vertices $\{1, \ldots, n\}$. Then, we say that $M$ is associated with $G$ if for any $p \in M$ there exists a neighbourhood $U(p)$ and a local orthonormal frame $\mathcal{B}=\left\{X_{1}, \ldots, X_{n}\right\}$ on $U$ satisfying the following conditions:

(a) $\left\{X_{1}, \ldots, X_{m}\right\}$ are tangent to $M$ and $\left\{X_{m+1}, \ldots, X_{n}\right\}$ are normal to $M$.

(b) For any $q \in U$, the graph $G_{\mathcal{B}, q}$ is isomorphic to $G$.

Example 3.1. As we have shown previously, every proper $\theta$-slant surface in a $4-$ dimensional almost Hermitian manifold is associated with Graph 2 of Figure 1. It is easy to prove that complex and totally real surfaces are associated with the same graph, by vanishing the corresponding edges when $\theta=0$ and $\theta=\pi / 2$, respectively. 
This situation can be generalized to slant submanifolds with arbitrary dimension by considering a general adapted slant frame (see [1 pp. 84-85]). Hence, we see that, in general, a $\theta$-slant submanifold is associated with the graph shown in Figure 2]

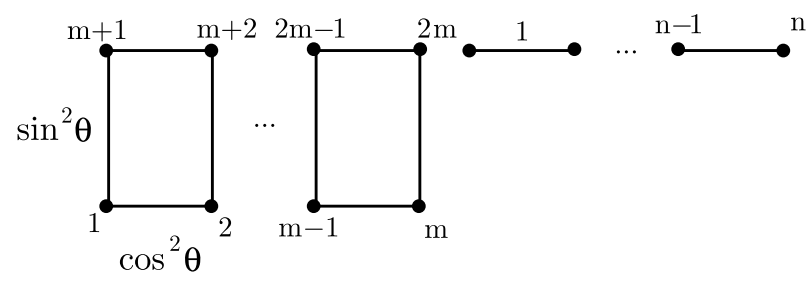

FiguRE 2. Slant submanifolds.

Example 3.2. By proceeding as in Example 3.1 we have semi-slant submanifolds associated with the graph shown in Figure 3

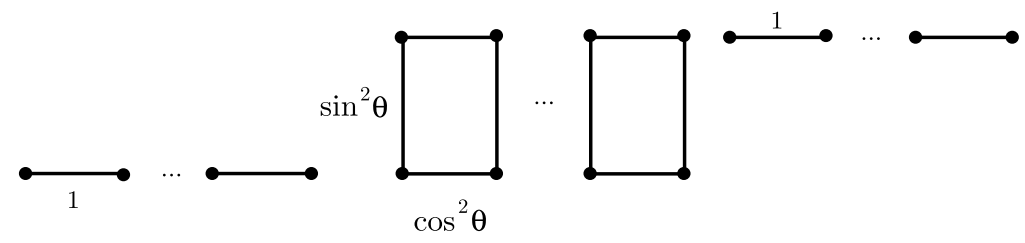

FIgURE 3. Semi-slant submanifolds.

Note that the above definition does depend on the chosen orthonormal frame. For example, if we consider a totally real surface $M^{2}$ in an almost Hermitian manifold $\widetilde{M}^{4}$, it is easy to prove that $M$ is associated with Graph 1 shown in Figure 4. In fact, it is enough to consider a local orthonormal frame $\left\{X_{1}, X_{2}, J X_{1}, J X_{2}\right\}$ on a neighbourhood of each point. Nevertheless, we can also take the frame

$$
\left\{\sqrt{2} / 2\left(X_{1}+X_{2}\right), \sqrt{2} / 2\left(X_{1}-X_{2}\right), J X_{1}, J X_{2}\right\} ;
$$

thus, $M$ is also associated with Graph 2 in Figure 4 (which is usally denoted by $\left.K_{2,2}\right)$.
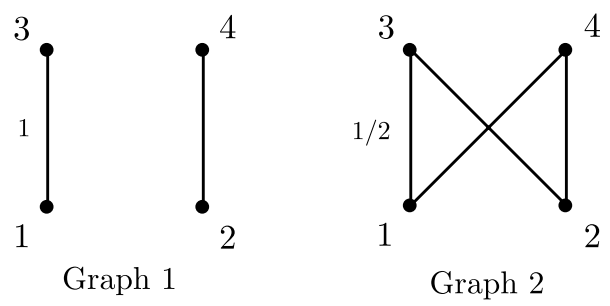

Figure 4. Totally real surface.

Now, we are going to show some general results which have to be satisfied by a graph in order for it to be associated with a submanifold. 
Lemma 3.3. Let $M$ be a submanifold associated with a graph $G$. Then, the sum of the weights of the incident edges on each vertex of $G$ must be equal to 1.

Proof. It follows directly from the compatibility between the metric and the almost complex structure on an almost Hermitian manifold.

Lemma 3.4. Let $M$ be a submanifold associated with a graph $G$. Then, $G$ has no isolated vertices.

Proof. Since $J^{2}=-I d$ on an almost Hermitian manifold, it follows that $J X \neq 0$ for any nonzero vector $X$. This implies Lemma 3.4

Proposition 3.5. Let $M$ be a submanifold associated with a graph $G$. Then, $G$ contains no isolated triangles.

Proof. Suppose that $G$ contains an isolated triangle, with vertices $k_{1}, k_{2}, k_{3}$. Let $A$ be the matrix with components $\left(g\left(J X_{i}, X_{j}\right)\right)$, where $\left\{X_{1}, \ldots, X_{n}\right\}$ is the frame given by the association between $M$ and $G$. Then, since $J^{2}=-I d$, it follows that $A^{2}=\left(a_{i j}\right)=-I$, where $I$ denotes the identity matrix. In particular, $a_{k_{1} k_{2}}=$ $0=\sum_{i=1}^{n} g\left(J X_{k_{1}}, X_{i}\right) g\left(J X_{i}, X_{k_{2}}\right)=g\left(J X_{k_{1}}, X_{k_{3}}\right) g\left(J X_{k_{3}}, X_{k_{2}}\right)$, which yields a contradiction.

Proposition 3.6. Let $M$ be a submanifold associated with a graph $G$. Let $v$ be a vertex in $G$ with degree 1 . Then, the connected component containing $v$ in $G$ is just a $K_{2}$.

Proof. Let $p \in M$ and consider the frame $\left\{X_{1}, \ldots, X_{n}\right\}$ on a neighbourhood of $p$ given by the definition of association between $M$ and $G$. We can suppose that a vertex $i$ has degree 1 and that it is adjacent to $j, j \neq i$. Hence, it follows from Lemma 3.3 that the weight of $\{i, j\}$ must be 1 . Therefore, there are no more vertices adjacent to $j$ and the proof is done.

By using this proposition, we can obtain the following characterization of CRsubmanifolds by means of trees (connected graphs without cycles) and forests (disjoint unions of trees; see [2]).

Theorem 3.7. A submanifold is associated with a forest if and only if it is a CR-submanifold. In this case, every tree is a $K_{2}$.

Proof. Suppose that a submanifold $M$ is associated with a forest $G$. By virtue of Proposition 3.6, every connected component of $G$ has to be a $K_{2}$, since every tree has vertices with degree 1 (see 2]). Then, we can suppose that the graph $G$ looks like that of Figure 5 , by reordering the local orthonormal frame given by the association if necessary, where $d_{1}+d_{2}=m$. Of course we consider $d_{1}=0$ (resp. $\left.d_{2}=0\right)$ if there are no tangent horizontal (resp. vertical) edges in $G$. Now, given $p \in M$, we can define $\mathcal{D}_{1}=\operatorname{Span}\left(X_{1}, \ldots, X_{d_{1}}\right)$ and $\mathcal{D}_{2}=\operatorname{Span}\left(X_{d_{1}+1}, \ldots, X_{d_{1}+d_{2}}\right)$ on a neighbourhood of $p$. It follows that $\mathcal{D}_{1}$ and $\mathcal{D}_{2}$ are two well-defined differentiable distributions on $M$ such that $T M=\mathcal{D}_{1} \oplus \mathcal{D}_{2}$. Moreover, $\mathcal{D}_{1}$ (resp. $\mathcal{D}_{2}$ ) is a complex (resp. totally real) distribution, and so, $M$ is a CR-submanifold.

The converse follows from a direct computation.

We have previously shown how this work was motivated by the study of slant surfaces. Now, we can prove that the association with graphs really characterizes this kind of submanifolds. 


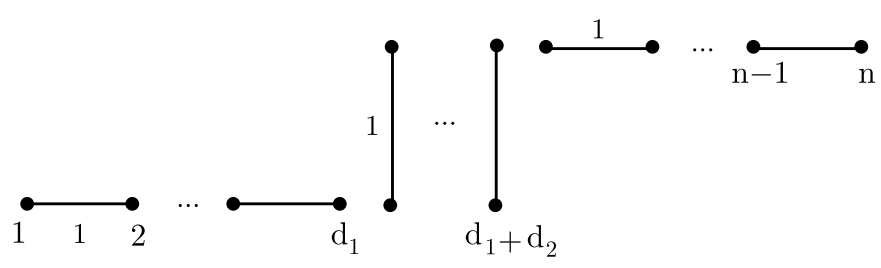

FiguRE 5. CR-submanifolds.

Theorem 3.8. Let $M^{2}$ be a surface isometrically immersed in an almost Hermitian manifold. Then, $M$ is slant if and only if there exists a graph $G$ such that $M$ is associated with $G$.

Proof. We already know that a slant surface is associated with a graph. Conversely, suppose that a surface $M$ is associated with a graph $G$. Let $i, j$ be the tangent vertices of $G$. If these vertices are not adjacent, then it is clear that $M$ is a totally real surface. Now, suppose that $i$ and $j$ are adjacent. If there are no other vertices adjacent to $i$ or $j$, then the weight of $\{i, j\}$ must be 1 and $M$ is a complex submanifold. If not, denote by $\mu$ the weight of $\{i, j\}, 0<\mu<1$. In this case, we have $P^{2}=-\mu I d$, thus $M$ is a slant submanifold with angle $\theta$ such that $\cos ^{2} \theta=\mu$.

\section{The 4-Dimensional CASE}

We now proceed to study submanifolds associated with graphs in a 4-dimensional almost Hermitian manifold $\widetilde{M}^{4}$. It is known that there are only 11 different graphs with 4 vertices [2]. But, by virtue of Lemma 3.3. Proposition 3.5] and Proposition 3.6 we can see that, if $M$ is a submanifold of $\widetilde{M}^{4}$ associated with a graph $G$, then $G$ must be one of the graphs drawn in Figure 6 .

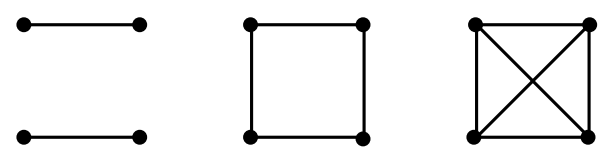

FIGURE 6. Submanifolds in a 4-dimensional space.

We have previously shown that, if a submanifold is associated with a graph, then this graph is not unique. Therefore, we now say that two weighted labeled graphs $G$ and $G^{\prime}$ are equivalent, $G \sim G^{\prime}$, if for any submanifold $M$ associated with $G$, then $M$ is associated with $G^{\prime}$ and for any submanifold $M^{\prime}$ associated with $G^{\prime}$, then $M^{\prime}$ is associated with $G$. Obviously, this is an equivalence relation on the class of graphs associated with submanifolds. Now our objective is to classify submanifolds associated with graphs through this relation.

We first consider curves in $\widetilde{M}$. It is easy to see that, if $M$ is a curve in $\widetilde{M}$ associated with a graph $G$, then, up to isomorphisms, $G$ must be one of the graphs from Figure 7 

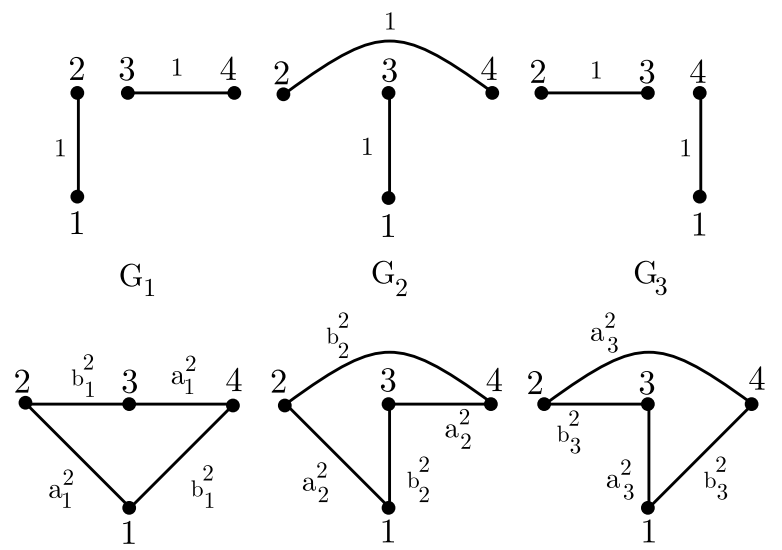

$\mathrm{G}_{4}$

$\mathrm{G}_{5}$

$\mathrm{G}_{6}$

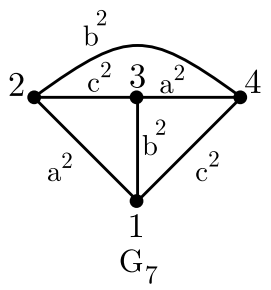

FiguRE 7. Graphs associated with curves.

We have the following result:

Theorem 4.1. All graphs shown in Figure 7 are equivalent.

Proof. We can easily see that graphs $G_{1}, G_{2}$ and $G_{3}$ are equivalent, simply by reordering the appropriate local orthonormal frames. Now, let us prove that $G_{1}$ is equivalent to $G_{4}\left(a^{2}, b^{2}\right)$, for any $a, b$ such that $a^{2}+b^{2}=1$. If $M$ is a submanifold associated with $G_{1}$ through a local frame $\left\{X_{1}, \ldots, X_{4}\right\}$ and we define the new orthonormal frame

$$
Y_{1}=X_{1}, \quad Y_{2}=a X_{2}+b X_{4}, \quad Y_{3}=X_{3}, \quad Y_{4}=b X_{2}-a X_{4},
$$

then, $M$ is associated with $G_{4}$ through $\left\{Y_{1}, \ldots, Y_{4}\right\}$. Conversely, it is enough to consider the change

$$
X_{1}=Y_{1}, \quad X_{2}=h_{1} Y_{2}+h_{2} Y_{4}, \quad X_{3}=Y_{3}, \quad X_{4}=h_{2} Y_{2}-h_{1} Y_{4},
$$

where $h_{1}=g\left(J Y_{1}, Y_{2}\right)$ and $h_{2}=g\left(J Y_{1}, Y_{4}\right)$. Similarly, we also have $G_{2} \sim G_{5}$ and $G_{3} \sim G_{6}$.

Finally, we show that $G_{4}\left(a^{2}+b^{2}, c^{2}\right) \sim G_{7}\left(a^{2}, b^{2}, c^{2}\right)$, for any $a, b, c$ such that $a^{2}+$ $b^{2}+c^{2}=1$. As above, if $\left\{X_{1}, \ldots, X_{4}\right\}$ (resp. $\left\{Y_{1}, \ldots, Y_{4}\right\}$ ) is a local orthonormal frame associated with $G_{4}\left(a^{2}+b^{2}, c^{2}\right)$ (resp. $\left.G_{7}\left(a^{2}, b^{2}, c^{2}\right)\right)$, then it is only necessary to take into account the changes

$$
Y_{1}=X_{1}, \quad Y_{2}=\lambda_{1} X_{2}+\mu_{1} X_{3}, \quad Y_{3}=\mu_{1} X_{2}-\lambda_{1} X_{3}, \quad Y_{4}=X_{4},
$$

with $\lambda_{1}^{2}=a^{2} /\left(a^{2}+b^{2}\right), \mu_{1}^{2}=b^{2} /\left(a^{2}+b^{2}\right)$ and

$$
X_{1}=Y_{1}, \quad X_{2}=\lambda_{2} Y_{2}+\mu_{2} Y_{3}, \quad X_{3}=\mu_{2} Y_{2}-\lambda_{2} Y_{3}, \quad X_{4}=Y_{4},
$$


with $\lambda_{2}=g\left(J Y_{1}, Y_{2}\right) / \sqrt{a^{2}+b^{2}}, \mu_{2}=g\left(J Y_{1}, Y_{3}\right) / \sqrt{a^{2}+b^{2}}$. Given that $\sim$ is an equivalence relation, the proof concludes.

Hence, we do not find different classes of curves associated with graphs. In particular, we see that any curve associated with a graph is a totally real submanifold. This is a general well-known fact for curves in almost Hermitian manifolds.

If we study surfaces in $\widetilde{M}$, the situation is quite different. The graphs associated with surfaces appear in Figure 8 . Denote by $\left[G_{i}\right]$ the equivalence class defined by $G_{i}$.
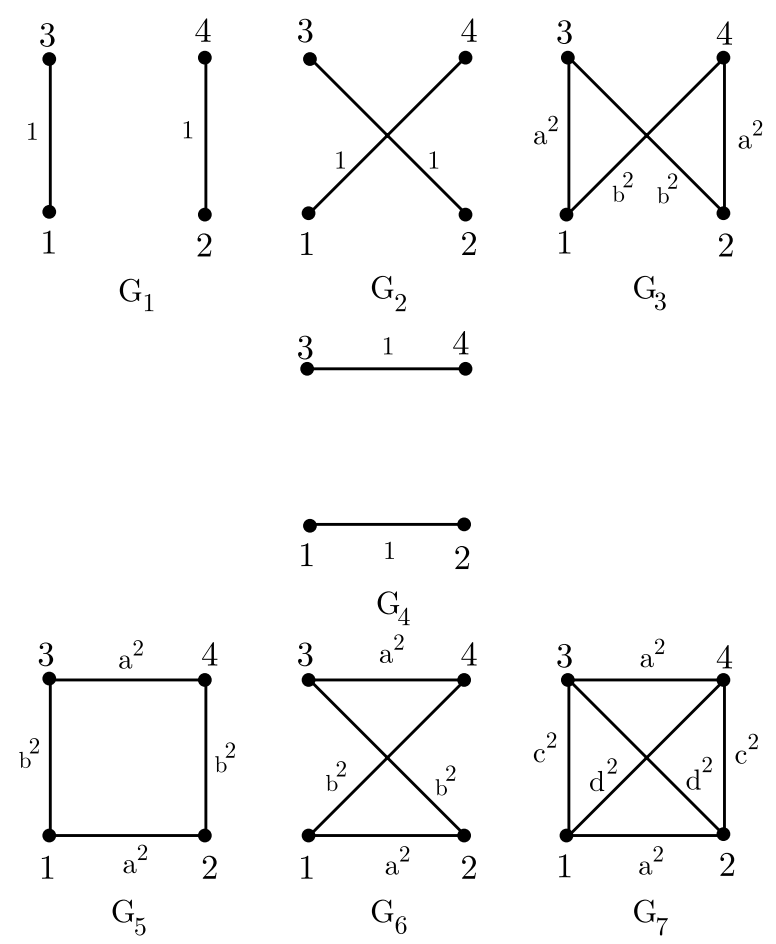

FiguRE 8. Graphs associated with surfaces.

Theorem 4.2. If we consider the graphs from Figure 8, then we have the following equivalence classes:

i) $\left[G_{1}\right]=\left[G_{2}\right]=\left[G_{3}\left(a^{2}, b^{2}\right)\right]$, for any $a, b \in \mathbf{R}$ such that $a^{2}+b^{2}=1$;

ii) $\left[G_{4}\right]$;

iii) for any $a^{2}>0$, we obtain a different equivalence class $\left[G_{5}\left(a^{2}, b^{2}\right)\right]=$ $\left[G_{6}\left(a^{2}, b^{2}\right)\right]=\left[G_{7}\left(a^{2}, c^{2}, d^{2}\right)\right]$, whichever $b, c, d \in \mathbf{R}$ are such that $a^{2}+b^{2}=$ $a^{2}+c^{2}+d^{2}=1$.

Moreover, given a surface $M^{2}$ in $\widetilde{M}^{4}, M$ is associated with the class $\left[G_{1}\right]$ (resp. $\left[G_{4}\right]$ ) if and only if it is a totally real surface (resp. complex surface), and $M$ is associated with $\left[G_{5}\left(a^{2}, b^{2}\right)\right]$ if and only if it is a proper slant surface with slant angle $\theta$ such that $a^{2}=\cos ^{2} \theta$. 


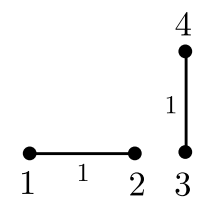

Figure 9. CR-Hypersurface.

Proof. The equivalence between graphs $G_{1}$ and $G_{2}$ (resp. $G_{5}$ and $G_{6}$ ) follows by reordering the corresponding frames. Let us now see that, given $a, b \in \mathbf{R}$ with $a^{2}+b^{2}=1$, then $G_{1}$ is equivalent to $G_{3}\left(a^{2}, b^{2}\right)$. If $M$ is a submanifold associated with $G_{1}$ through a local frame $\left\{X_{1}, \ldots, X_{4}\right\}$ and we define the new orthonormal frame

$$
Y_{1}=a X_{1}+b X_{2}, \quad Y_{2}=b X_{1}-a X_{2}, \quad Y_{3}=X_{3}, \quad Y_{4}=X_{4},
$$

then, $M$ is associated with $G_{3}\left(a^{2}, b^{2}\right)$ through $\left\{Y_{1}, \ldots, Y_{4}\right\}$. Conversely, suppose that $M^{\prime}$ is a submanifold associated with $G_{3}\left(a^{2}, b^{2}\right)$ through a local frame $\left\{Y_{1}, \ldots, Y_{4}\right\}$. Hence, we can first deduce from $J^{2}=-I d$ that

$$
\begin{gathered}
g\left(J Y_{1}, Y_{3}\right) g\left(J Y_{2}, Y_{4}\right)-g\left(J Y_{1}, Y_{4}\right) g\left(J Y_{2}, Y_{3}\right)= \pm 1, \\
g\left(J Y_{1}, Y_{3}\right) g\left(J Y_{1}, Y_{4}\right)+g\left(J Y_{2}, Y_{3}\right) g\left(J Y_{2}, Y_{4}\right)=0 .
\end{gathered}
$$

Then, to prove that $M^{\prime}$ is associated with $G_{1}$, it is enough to consider the change

$$
X_{1}=-h_{1} Y_{1}+h_{2} Y_{2}, \quad X_{2}=h_{2} Y_{1}+h_{1} Y_{2}, \quad X_{3}=Y_{3}, \quad X_{4}=Y_{4},
$$

where $h_{1}=g\left(J Y_{2}, Y_{4}\right)$ and $h_{2}=g\left(J Y_{1}, Y_{4}\right)$.

Given $a, b, c, d \in \mathbf{R}$ with $a^{2}+b^{2}=a^{2}+c^{2}+d^{2}=1$, to prove that $G_{5}\left(a^{2}, b^{2}\right)$ and $G_{7}\left(a^{2}, c^{2}, d^{2}\right)$ are equivalent, we only have to take into account the changes

$$
Y_{1}=\lambda_{1} X_{1}+\mu_{1} X_{2}, \quad Y_{2}=\mu_{1} X_{1}-\lambda_{1} X_{2}, \quad Y_{3}=X_{3}, \quad Y_{4}=X_{4},
$$

with $\lambda_{1}^{2}=c^{2} / b^{2}, \mu_{1}^{2}=d^{2} / b^{2}$ and

$$
X_{1}=-\lambda_{2} Y_{1}+\mu_{2} Y_{2}, \quad X_{2}=\mu_{2} Y_{1}+\lambda_{2} Y_{2}, \quad X_{3}=Y_{3}, \quad X_{4}=Y_{4},
$$

with $\lambda_{2}=g\left(J Y_{2}, Y_{4}\right) / b, \mu_{2}=g\left(J Y_{1}, Y_{4}\right) / b$, where $\left\{X_{1}, \ldots, X_{4}\right\}$ (resp. $\left.\left\{Y_{1}, \ldots, Y_{4}\right\}\right)$ is a local orthonormal frame associated with $G_{5}\left(a^{2}, b^{2}\right)$ (resp. $\left.G_{7}\left(a^{2}, c^{2}, d^{2}\right)\right)$.

Finally, it is easy to see that $G_{1}, G_{4}$ and $G_{5}$ are not equivalent to each other, since they represent totally different behaviours of a submanifold with respect to the almost complex structure $J$. Hence, we finish this proof by following that of Theorem 3.8 .

In this case, we see that the classification of graphs also classifies surfaces associated with graphs attending to their behaviour with respect to the almost complex structure of $\widetilde{M}$. We have found different classes of surfaces: totally real surfaces, complex surfaces and proper slant surfaces. In fact, we have proven that every surface associated with a graph in a 4 -dimensional almost Hermitian manifold is a slant surface, which is a particular case of Theorem 3.8 .

Finally, we see that the situation for hypersurfaces associated with graphs looks like that of curves. We can prove the corresponding similar theorem:

Theorem 4.3. All graphs associated with hypersurfaces of $\widetilde{M}^{4}$ are equivalent. 
It is clear that a submanifold associated with the graph shown in Figure 9 is a CR-submanifold. So, it follows from Theorem 4.3 that each hypersurface of $\widetilde{M}^{4}$ associated with a graph is a CR-submanifold.

\section{REFERENCES}

1. B.-Y. Chen, Geometry of slant submanifolds, Katholieke Universiteit Leuven, Louvain, 1990. MR 92d:53047

2. F. Harary, Graph theory, Addison-Wesley, Reading, Mass., 1969. MR 41:1566

3. N. Papaghiuc, Semi-slant submanifolds of a Kaehlerian manifold, An. Ştiinţ. Univ. Al. I. Cuza Iaşi. 40 (1994), 55-61. MR 96d:53066

4. K. Yano and M. Kon, Structures on manifolds, Series in Pure Mathematics 3, World Scientific, Singapore, 1984. MR 86g:53001

Department of Geometry and Topology, Faculty of Mathematics, University of Sevilla, Apdo. Correos 1160, 41080-Sevilla, Spain

E-mail address: carriazo@us.es

Department of Geometry and Topology, Faculty of Mathematics, University of Sevilla, Apdo. Correos 1160, 41080-Sevilla, Spain

E-mail address: Imfer@us.es 